\title{
Splitting of the Magnetic Loss Peak of Composites under External Magnetic Field
}

\author{
Artem Shiryaev *, Konstantin Rozanov, Andrey Naboko, Anastasia Artemova, Sergey Maklakov (D), \\ Stanislav Bobrovskii and Dmitriy Petrov
}

check for

updates

Citation: Shiryaev, A.; Rozanov, K.; Naboko, A.; Artemova, A.; Maklakov, S.; Bobrovskii, S.; Petrov, D. Splitting of the Magnetic Loss Peak of Composites under External Magnetic Field. Physics 2021, 3, 678-688.

https: / / doi.org/10.3390/ physics3030041

Received: 30 June 2021

Accepted: 18 August 2021

Published: 23 August 2021

Publisher's Note: MDPI stays neutral with regard to jurisdictional claims in published maps and institutional affiliations.

Copyright: (c) 2021 by the authors. Licensee MDPI, Basel, Switzerland. This article is an open access article distributed under the terms and conditions of the Creative Commons Attribution (CC BY) license (https:/ / creativecommons.org/licenses/by/ $4.0 /)$.
Institute for Theoretical and Applied Electromagnetics, Moscow 125412, Russia; k.rozanov@yandex.ru (K.R.); nas.webwork@gmail.com (A.N.); kosevich96@yandex.ru (A.A.); squirrel498@gmail.com (S.M.); sbobrowskiy@yandex.ru (S.B.); dpetrov-itae@yandex.ru (D.P.)

* Correspondence: artemshiryaev@mail.ru

Abstract: Composite materials filled with ferromagnetic inclusions are useful in the development of various microwave devices. The performance of such devices is determined both by material properties (such as the saturation magnetization and the permeability) and by the demagnetization effects. The paper is devoted to the study of the demagnetization effect on the permeability measurements of composites under external magnetic bias. The microwave permeability of composites filled with flake sendust (Fe-Si-Al alloy) particles is measured as a function of frequency and the external magnetic field. The measurements are carried out by the Nicolson-Ross-Weir technique in a $7 / 3$ coaxial line in the frequency range of 0.1 to $20 \mathrm{GHz}$ by a vector network analyzer. It is found that the magnetic loss peak is split under external fields of more than $1.5 \mathrm{kOe}$. The main aim of this paper is to study the causes of this splitting and to interpret the observed magnetic loss peaks. To study this effect, the samples of various thicknesses and the samples with isotropic and anisotropic orientations of particles are measured. The particles in the anisotropic samples are oriented by a strong uniform magnetic field. At a small fraction of inclusions, the permanent magnetic field is demagnetized on the individual particles rather than the whole sample. The splitting of the magnetic loss peak of the isotropic sample is caused by different orientations of particles in the sample. At a high fraction of inclusions, the permanent magnetic field is demagnetized on the whole sample and the magnetic loss peak of the isotropic sample is not split. The saturation magnetization of the material is found by measurements under the external magnetic field of the anisotropic sample.

Keywords: composites; ferromagnetic resonance; microwave permeability; demagnetization

\section{Introduction}

Composites filled with ferromagnetic inclusions possess a combination of promising magnetic, electrical, optical, and mechanical properties. High-frequency magnetic properties of the composites are useful in the development of various microwave devices [1-5]. The performance of microwave devices is dependent on both the material properties (such as the saturation magnetization and the permeability) and the demagnetization effects [6-9].

The microwave spectrum of permeability of composites may have a complicated shape comprising several magnetic loss peaks. The reliable techniques for the interpretation of the peaks are not found in the archived literature. The standard ferromagnetic resonance (FMR) technique [10-12] makes it possible to find the magnetic characteristics of materials, but the observed peaks may not be interpreted. Moreover, extraneous resonances can be observed in addition to FMR, the appearance of which is difficult to explain [13].

Many studies concerned with developments in microwave electronics insist on the important role played by the demagnetizing effects on devices [6-9]. In the case of composites, the influence of demagnetization effects depends not only on the shape of the device 
but also on the concentration of the ferromagnetic inclusions in the composite. The effect of demagnetization on composites was studied in [14-16].

Thus, for the use of composites in microwave devices, knowledge of both demagnetizing effects and the physical mechanisms resulting in the appearance of the magnetic loss peaks is important.

The technique proposed in $[17,18]$ may be used to study demagnetization effects, to interpret the magnetic loss peaks, and to determine the saturation magnetization. The technique represents the coaxial measurements of complex permeability depending both on frequency, $f$, and on external field, $H: \mu(f, H)=\operatorname{Re}[\mu(f, H)]+\mathrm{i} \cdot \operatorname{Im}[\mu(f, H)]$. The measuring coaxial cell is placed inside a coil that creates magnetic bias parallel to the coaxial axis.

The measurements under various permanent magnetic fields provide an opportunity to compare the magnetic characteristics of a sample in both the demagnetized and magnetized states, determine the physical mechanisms responsible for different peaks of magnetic loss, and find the magnetic characteristics of the material.

The aim of the paper is to study the effect of demagnetization on the microwave permeability of composites and to interpret observed magnetic loss peaks by this technique. The samples under study are composites filled with flake-shaped sendust $\left(\mathrm{Al}_{0.054} \mathrm{Si}_{0.096} \mathrm{Fe}_{0.85}\right)$ particles.

It is found that the magnetic loss peak of isotropic composite is split under external fields of more than $1.5 \mathrm{kOe}$. To search for the reasons for the peak splitting, the demagnetizing effects are studied on the samples with different thicknesses and samples with an anisotropic distribution of inclusions. Measurements of the samples with an anisotropic orientation of particles may provide important data on the features of the demagnetization of composite materials.

\section{Materials and Methods}

The samples under study are composites with a paraffin wax matrix filled with flakeshaped sendust $\left(\mathrm{Al}_{0.054} \mathrm{Si}_{0.096} \mathrm{Fe}_{0.85}\right)$ particles. The flake particles were obtained by spraying a molten sendust alloy and grinding the resulting particles with a ball mill. Particles with a size of 40 to $63 \mu \mathrm{m}$ were obtained by sieving. The thickness of the particles was about 1-2 $\mu \mathrm{m}$. SEM (scanning electron microscope) microphotos of the particles and the curve of particle size distribution are presented in [19]. The samples were made by mixing sendust powder and melted paraffin wax. Mixing was continued until the solidification of the mixture. The resulting mixture was pressed into the coaxial sample with an outer diameter of $7 \mathrm{~mm}$ and an inner diameter of $3 \mathrm{~mm}$. The samples with a volume concentration of inclusions of 2 to 12 vol.\% were made. The concentration of inclusions was found from the thickness of the sample and the masses of paraffin, filler, and the obtained sample. The volume concentration of air pores was less than $3 \%$.

An SEM microphoto of the sample with $4 \%$ volume fraction of inclusions is shown in Figure 1. The sample contains clusters of particles. They are marked in red in the figure. It is seen that the inclusions are randomly distributed in all directions and the sample may be considered isotropic.

Anisotropic composites were made from isotropic samples with a $4 \%$ volume fraction of inclusions. The isotropic samples were placed in a sealed coaxial mold. The mold with the sample was heated to $100^{\circ} \mathrm{C}$ for one minute until the paraffin wax melts. During the heating process, the mold was slowly rotated to avoid the formation of particle clusters due to the influence of gravity. Then, the mold with the molten sample was placed inside the electromagnet, and the magnetic field $\mathrm{H}_{\text {ext }}$ was increased up to $10 \mathrm{kOe}$. The mold was also rotated inside the electromagnet for five minutes until the paraffin solidified in the sample. 


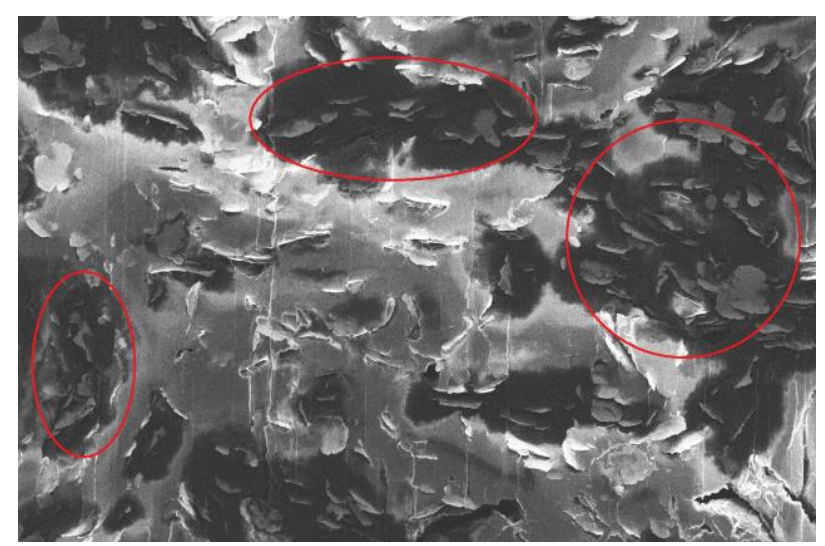

Figure 1. SEM (scanning electron microscope) photos of sections of the studied composite samples. Volume fraction of inclusions in the sample is $4 \%$.

Two anisotropic samples with different particle distributions were prepared. The first sample was placed and rotated in the electromagnet as shown in Figure 2a. With this orientation of the sample, the external field is perpendicular to the coaxial axis, and the flake-shaped particles in the sample are oriented in the plane perpendicular to the coaxial axis. The position of the second sample in the electromagnet is shown in Figure 2b: the magnetic field is directed along the coaxial axis; rotation was also carried out around this axis. In this case, planar particles can be oriented in all planes parallel to the coaxial axis. In both samples, the volume fraction of inclusions is $4 \%$.

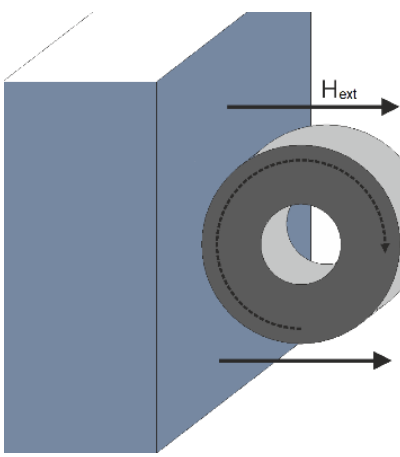

(a)

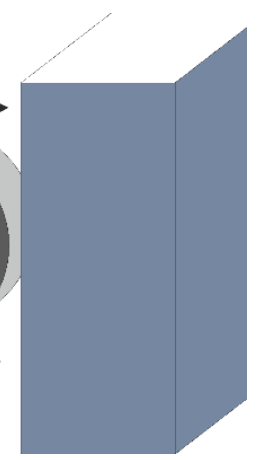

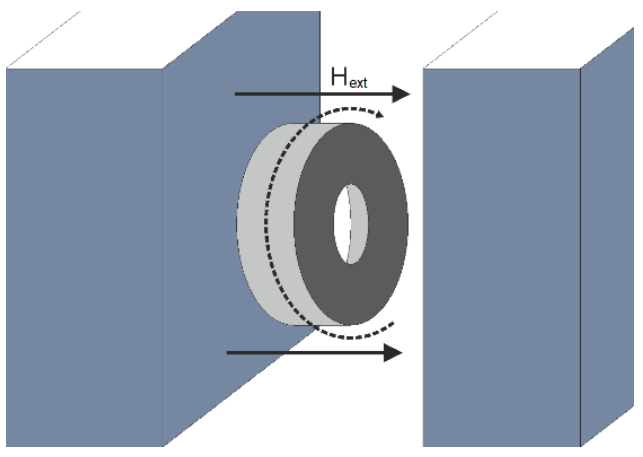

(b)

Figure 2. Position of coaxial anisotropic samples inside the electromagnet: (a) particles in the resulting sample are oriented in a plane perpendicular to the coaxial axis; (b) particles are oriented along the coaxial axis. The solid arrows indicate the direction of the magnetic field $\mathrm{H}_{\mathrm{ext}}$ of the electromagnet.

The frequency dependencies of microwave permittivity and permeability of the samples were measured by the Nicolson-Ross-Weir $[19,20]$ (NRW) technique in a $7 / 3$ coaxial line in the frequency range of 0.1 to $20 \mathrm{GHz}$ by a vector network analyzer. The measurement cell was placed inside a coil producing a static magnetic field $\mathrm{H}_{\text {bias }}$ up to 2400 Oe. The permanent magnetic field was directed parallel to the coaxial axis and perpendicular to the microwave magnetic field. Each sample was additionally pressed in the measuring cell in order to reduce the gaps between the sample and the walls of the cell. This allowed the error in the measured permittivity to be diminished.

\section{Theoretical Background}

In the absence of an external magnetic field, the magnetic moments of the ferromagnetic particles of the composites are oriented along the anisotropy fields, which are randomly distributed in directions. An increase in the external magnetic field leads to the disappearance of the domain structure, the rotation of the magnetization vectors in 
the direction of the external field, and a decrease in the inhomogeneous distribution of magnetization vectors in the material. If the sample is magnetized to saturation (the magnetic moments of all particles are directed along the external field), the permeability obeys the theory of Landau-Lifshitz-Gilbert (LLG) [21,22] and Kittel [23]. It is expected that only the magnetic loss peak associated with the uniform ferromagnetic resonance remains. This behavior was observed both in thin ferromagnetic films [24,25] and in composite materials [26]. The resonance frequency of platelet particles and thin ferromagnetic films depends on the external field according to Kittel's law:

$$
f_{\text {res }}=\gamma \sqrt{\left(H_{\mathrm{k}}+H_{\text {bias }}\right)\left(H_{\mathrm{k}}+H_{\text {bias }}+4 \pi M_{0}\right)},
$$

where $\gamma$ is the gyromagnetic ratio, $H_{\mathrm{k}}$ is the anisotropy field, $H_{\text {bias }}$ is the external magnetic field, $4 \pi M_{0}$ is the saturation magnetization, and $f_{\text {res }}$ is the resonant frequency. The anisotropy field and the saturation magnetization are often found by the FMR technique and Kittel's law (1) $[27,28]$. Measurements of the microwave permeability under a magnetic field can also be used to determine these magnetic characteristics [17].

The permeability associated with the ferromagnetic resonance is described by the LLG dispersion law [29]. In the case of thin films and platelet inclusions, the difference between LLG and Lorentzian dispersion laws is insignificant [21]. The LLG parameters may be obtained from the parameters of Lorentzian law [29]. Therefore, one describes the microwave permeability by the Lorentzian law [30-32]:

$$
\mu(f)=\mu_{\infty}+\sum_{j=1}^{n}\left(\mu_{\mathrm{st}, j}-1\right) /\left[1+i f / f_{\mathrm{rel}, j}-\left(f / f_{\mathrm{res}, j}\right)^{2}\right],
$$

where $\mu_{\infty}$ is the optical permeability equal to unity in most magnetic materials, $\mu_{\mathrm{st}}$ is the static permeability, $f_{\text {rel }}$ is the relaxation frequency, $f_{\text {res }}$ is the resonant frequency, and $n$ is the number of resonances.

\section{Results and Discussion}

The measured permeability of the composite sample with $7 \%$ volume fraction of inclusions under an external magnetic field up to 2400 Oe is shown in Figure 3. The resonant frequency increases and the static permeability decreases with increasing external field. The dependences are fitted by the LLG and Lorentzian dispersion laws. The fitting curves are almost identical, and the figure shows only the dependences with Lorentzian fitting. One can see that the magnetic loss peak has a Lorentzian form: the permeability is fitted by the dispersion law with high accuracy.

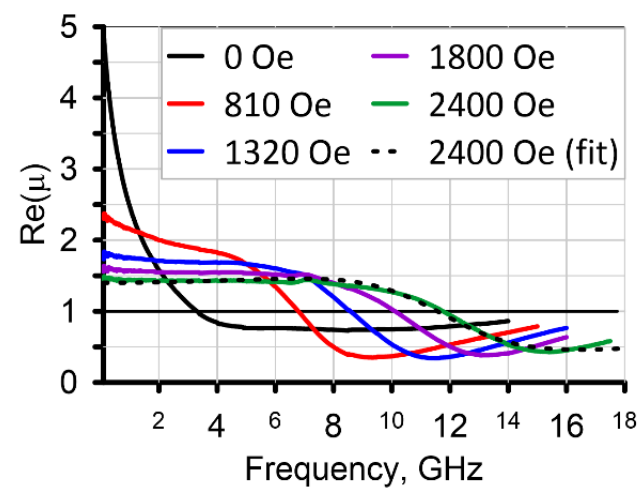

(a)

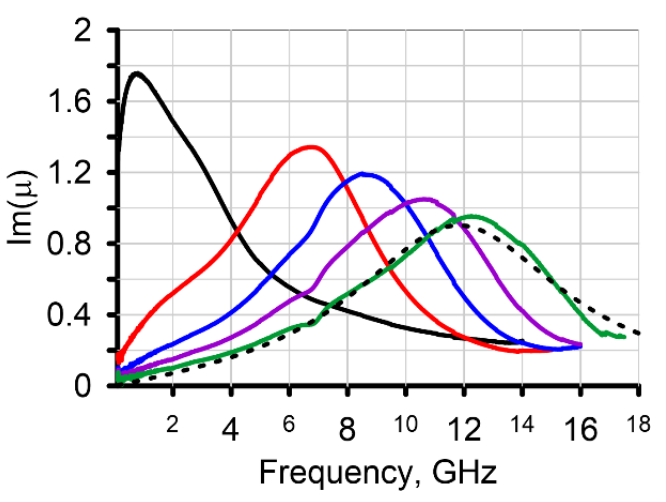

(b)

Figure 3. The measured dependences of the real (a) and imaginary (b) parts of permeability with volume fraction of inclusions of $7 \%$ under an external magnetic field up to 2400 Oe. The dashed line corresponds to the fitting by the Lorentzian dispersion law. 
The measured permeability of the composite sample with a lower volume fraction of inclusions (4\%) under an external magnetic field up to 2400 Oe is shown in Figure 4. It is seen that the peak becomes asymmetric under external field of more than 1350 Oe. The fitting of the permeability (dashed line in Figure 4) shows that the peak is not described by either the Lorentzian or LLG dispersion laws. However, the measured permeability is fitted by two Lorentzian curves (dotted line in Figure 4) with high accuracy. An increase in the external magnetic field leads to a divergence of these peaks. Therefore, the magnetic loss peak is split in the magnetic field. A similar splitting is also observed in the permeability of the samples with $2 \%$ and $3 \%$ fractions of inclusions.

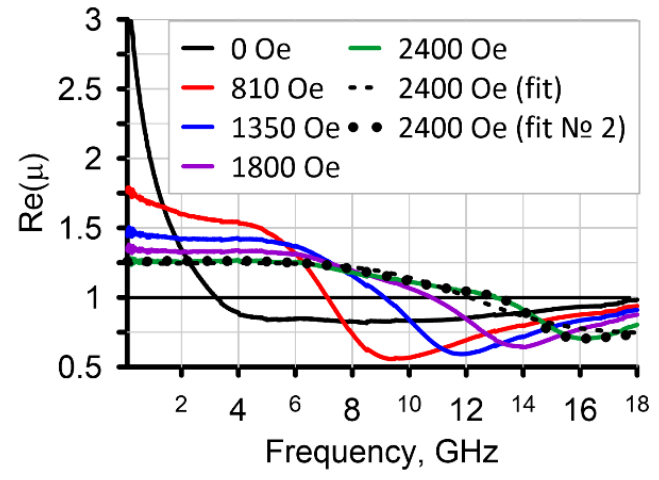

(a)

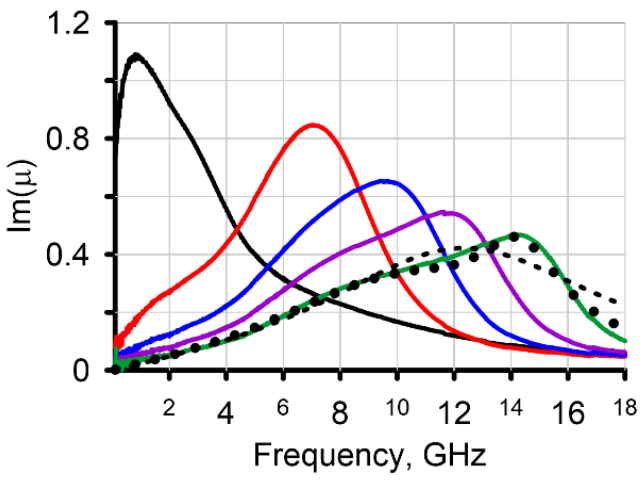

(b)

Figure 4. The measured dependences of the real (a) and imaginary (b) parts of permeability with volume fraction of inclusions of $4 \%$ under an external magnetic field up to 2400 Oe. The dashed line corresponds to the fitting by the Lorentzian dispersion law. The dotted line corresponds to the fitting by two Lorentzian curves.

The nature of these peaks and the physical mechanisms leading to the splitting are unknown. It is incorrect to find the magnetic characteristics of these peaks using Kittel's law (1). It is known that the skin effect may lead to the splitting of the magnetic loss peaks [33], but it is unclear why the splitting disappears with an increase in the fraction of inclusions.

The reason for the splitting of the magnetic loss peak under an external magnetic field may be the different magnetization of particles with various orientations in the sample. Demagnetization leads to a decrease in the external magnetic field inside the particle and is described by [14]:

$$
H=H_{\text {bias }} /\left[1+4 \pi \mu\left(\mu_{\text {st }}-1\right) N\right]
$$

where $H$ is the value of the external field decreased due to the demagnetization, $\mu_{\mathrm{st}}$ is the static permeability in the direction of the external field, and $N$ is the demagnetization factor of the particle in the direction of the external field. In the cases of a platelet particle and thin film, the demagnetization factor in the direction perpendicular to the plane is close to unity, and the other two factors are close to zero. Demagnetization has a stronger effect on particles oriented perpendicular to the external field. The magnetic field required to magnetize such particles is much greater than for the particles oriented along the field. In the composites under study, particles are distributed isotropically and differently oriented particles are magnetized variously. The FMR peak should broaden with the increasing external field. At high fractions of inclusions, the particles are located closer to each other, and the external field is demagnetized not on individual particles but on the whole sample. Therefore, the effect of peak splitting disappears at high fractions of inclusions in composites. It was shown in [15] that the demagnetization factor has a percolative behavior in composites. If the fraction of inclusions is greater than a certain value, the external field is demagnetized on the bulk sample. 
The effect of the demagnetization is studied on the isotropic samples with various thicknesses. The measured permeability of samples with different thicknesses and fractions of inclusion is shown in Figure 5. The permeability of the samples with a $4 \%$ fraction of inclusions does not depend on the thickness of the samples, see Figure 5a. The permeability of the samples with a $7 \%$ fraction of inclusions is also the same for different thicknesses. Samples with $9 \%, 10 \%$, and $12 \%$ fraction of inclusions were used. A noticeable discrepancy in the permeability under external field is observed at $12 \%$ samples, see dashed curves in Figure $5 \mathrm{~b}$. The measurement technique is sensitive to the thickness and permeability of the samples. In the case of thin samples, the measurement sensitivity may be poor, and they are also difficult to make. The measurement results of samples with a large thickness are strongly distorted by higher-order modes [34]. Therefore, the samples of only two thicknesses are shown.

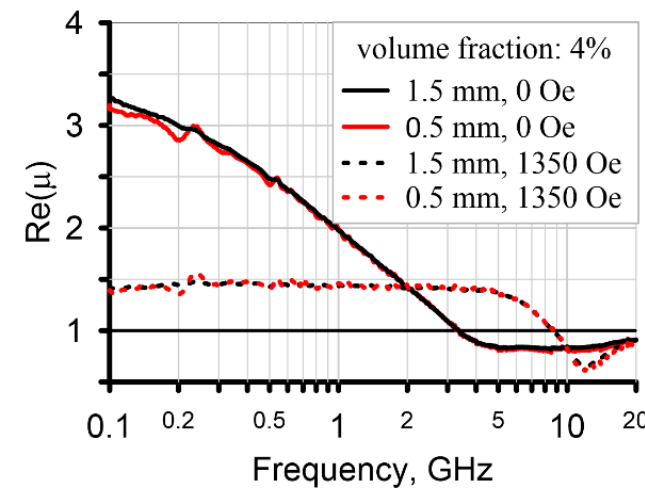

(a)

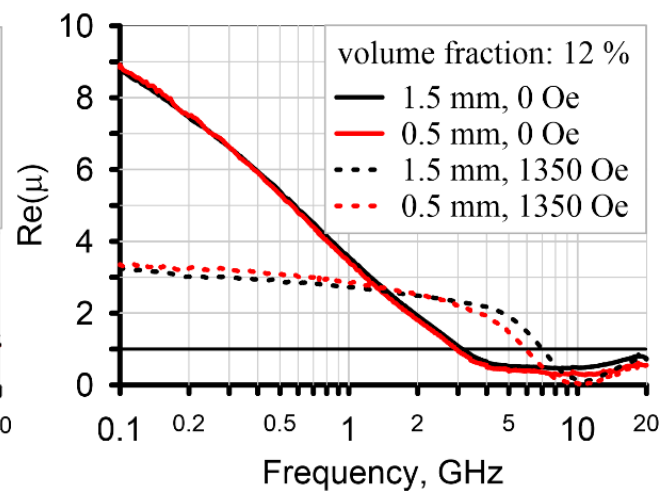

(b)

Figure 5. The measured frequency dependences of the permeability for the samples with different thicknesses and volume fractions of inclusion of $4 \%(\mathrm{a})$ and $12 \%(\mathrm{~b})$. The red curves correspond to the samples with a thickness of $0.5 \mathrm{~mm}$, and the black curves correspond to the samples with a thickness of $1.5 \mathrm{~mm}$. Dashed lines correspond to the data measured under the external magnetic field.

Thus, the permanent magnetic field is demagnetized on the individual particles at a small fraction of inclusions. An increase in the fraction leads to a decrease in the average distance between inclusions, and the external field is demagnetized on the whole sample. According to Equation (3), thicker samples are more strongly magnetized because they have a lower demagnetization factor. In the fraction range of $7 \%$ to $12 \%$, demagnetization may occur on clusters of inclusions. The obtained results may be quantitatively different for other shapes and materials of inclusions in the composite. Previously, a qualitatively similar behavior was observed in [15].

The samples with anisotropic particle distribution are prepared to test this hypothesis. The technique for the manufacture of anisotropic samples was described in Section 2. In the first and second anisotropic samples, the particles are oriented perpendicular and parallel to the coaxial axis, respectively. The constitutive parameters of the manufactured anisotropic samples are measured by the NRW technique under an external magnetic field of up to 2400 Oe. Figure 6 shows the frequency dependences of the permittivity and permeability of anisotropic and isotropic samples with the same volume fraction of inclusions of $4 \%$ under zero external field. The measured permittivity at high frequencies is distorted due to the influence of higher-order modes [34]. 

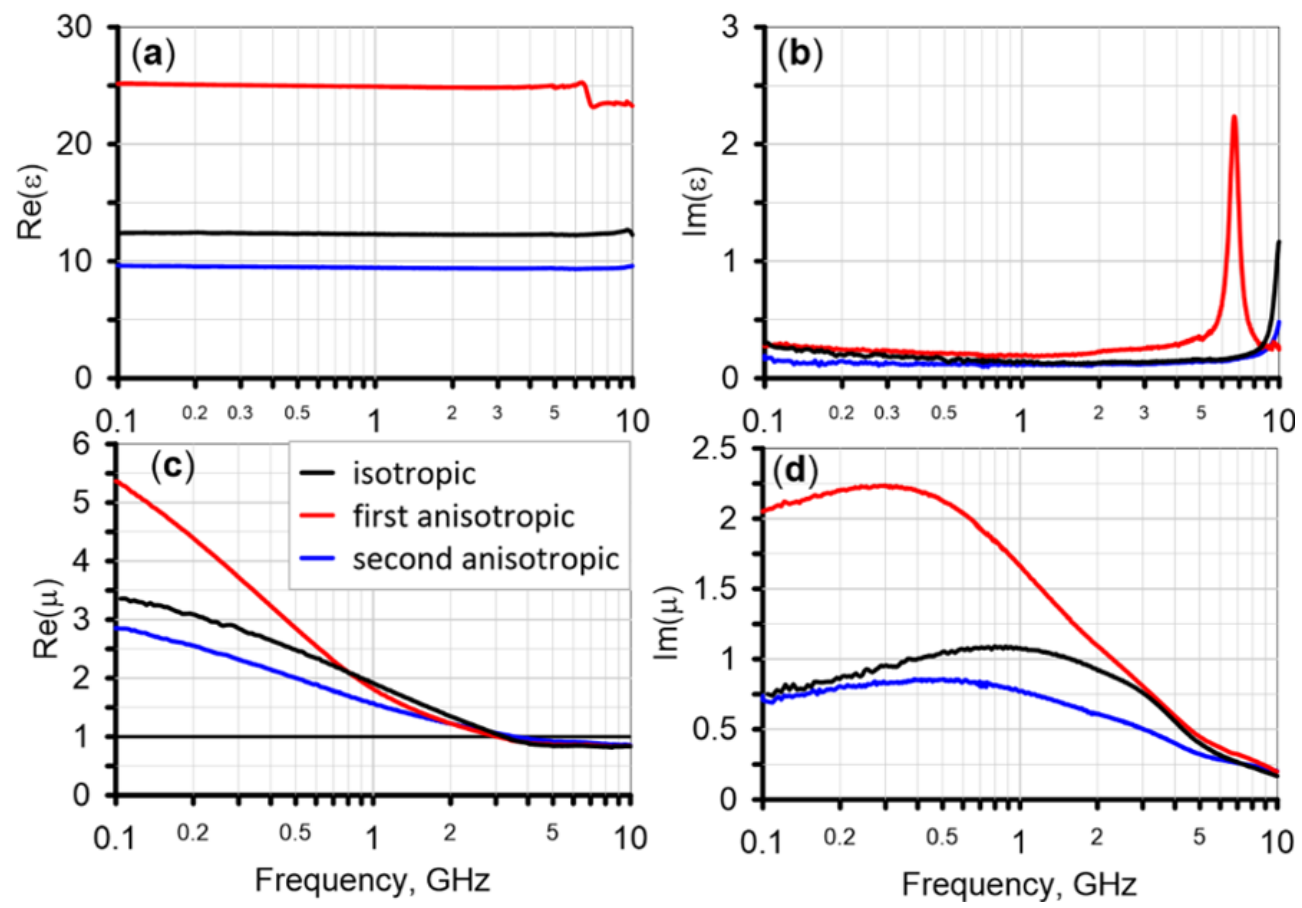

Figure 6. The measured frequency dependences of the real (a) and imaginary (b) parts of permittivity and the real (c) and imaginary (d) parts of permeability of the samples with $4 \%$ volume fraction of inclusions. Black curves correspond to the dependence for the isotropic sample. The red curves correspond to the dependence for the anisotropic sample with particles oriented perpendicular to the coaxial axis (the first anisotropic sample). The blue curves correspond to the dependence for the anisotropic sample with particles oriented parallel to the coaxial axis (the second anisotropic sample).

The largest contribution to the measured permittivity is made by platelet particles oriented perpendicular to the coaxial axis. The fraction of such particles in the isotropic sample may be estimated as $k=2 / 3$, in the first anisotropic sample as $k=1$, and in the second as $k=1 / 2$.

If the easy axes of magnetization are oriented in the plane of the particles, only one of the three components contributes to the measured permeability. For isotropic distribution of magnetic moments in space, $k=1 / 3$, and for isotropic distribution in the plane, $k=1 / 2$ [35]. For the second anisotropic sample, $k=1 / 4$. In the multidomain particles, the values of $k$ are the same as for the permittivity.

It is seen that the maximum of magnetic loss in the anisotropic samples is shifted to low frequencies. The reason for shifting may be explained by the following factors: incomplete anisotropy of the samples, an increase in the interaction between inclusions, and clustering due to inhomogeneity of the magnetic field.

The first anisotropic sample has large permittivity and permeability in comparison with the isotropic sample. The second sample has lower constitutive parameters. The observed behavior of the constitutive parameters is in qualitative agreement with the theory. Note that the use of composites with anisotropic particle distribution may be useful for practical applications [36]. The first anisotropic sample has higher values of microwave magnetic loss and static permeability compared to the isotropic sample with the same weight.

The measured permeability of the first anisotropic sample under an external field is shown in Figure 7, and the permeability of the second sample is shown in Figure 8. The demagnetization factor of the particles of the first sample in the direction of the external field is close to the unity. Because of this, the first anisotropic sample is magnetized weaker than the isotropic one. In the second anisotropic sample, in contrast, the demagnetization factor of the particles is close to zero, and the sample is magnetized more strongly than 
the isotropic one. The splitting of the magnetic loss peak observed for the isotropic sample is absent for the anisotropic samples. Note that the first anisotropic sample may not be magnetized to such a state that a noticeable splitting of the peak is observed.

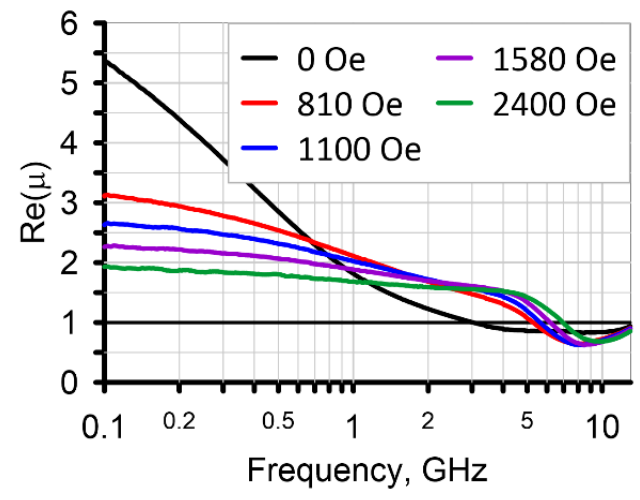

(a)

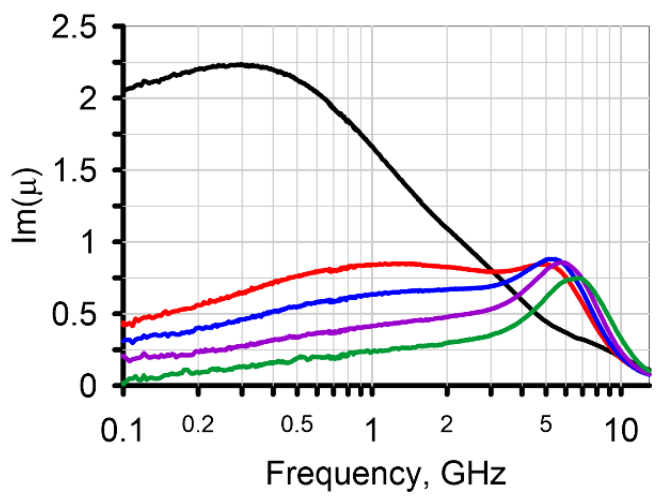

(b)

Figure 7. The measured dependences of the real (a) and imaginary (b) parts of permeability of the first anisotropic sample under an external magnetic field of up to 2400 Oe.

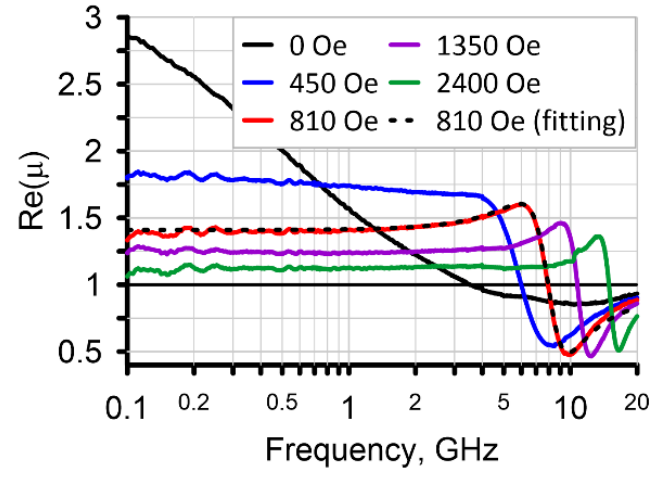

(a)

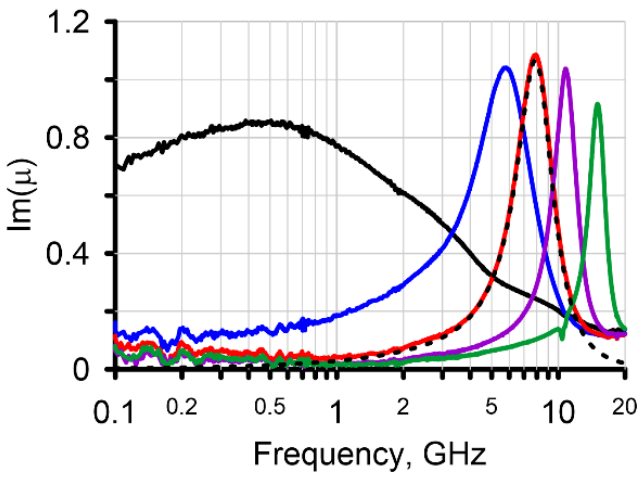

(b)

Figure 8. The measured dependences of the real (a) and imaginary (b) parts of permeability of the second anisotropic sample under external magnetic field up to 2400 Oe. The dashed line corresponds to the fitting by the Lorentzian dispersion law.

Thus, the influence of demagnetizing fields on the first anisotropic sample is maximal, and on the second sample, it is minimal. In the isotropic sample, the effect of demagnetizing fields is different depending on the orientation of a particular inclusion. This effect leads to the observed splitting of the peak. The frequency dependences of the permeability of the isotropic sample are fitted by the Lorentzian dispersion law with two terms. The dependences of the resonant frequencies on the applied field obtained from this fitting are shown in Figure 9 by red and green circles. The dependences for the first and the second anisotropic samples are also shown in Figure 9 by blue and black circles, respectively. It is seen that the frequencies of the isotropic sample are between the frequencies of the anisotropic ones. This behavior is associated with the random orientation of the particles in the isotropic sample. 


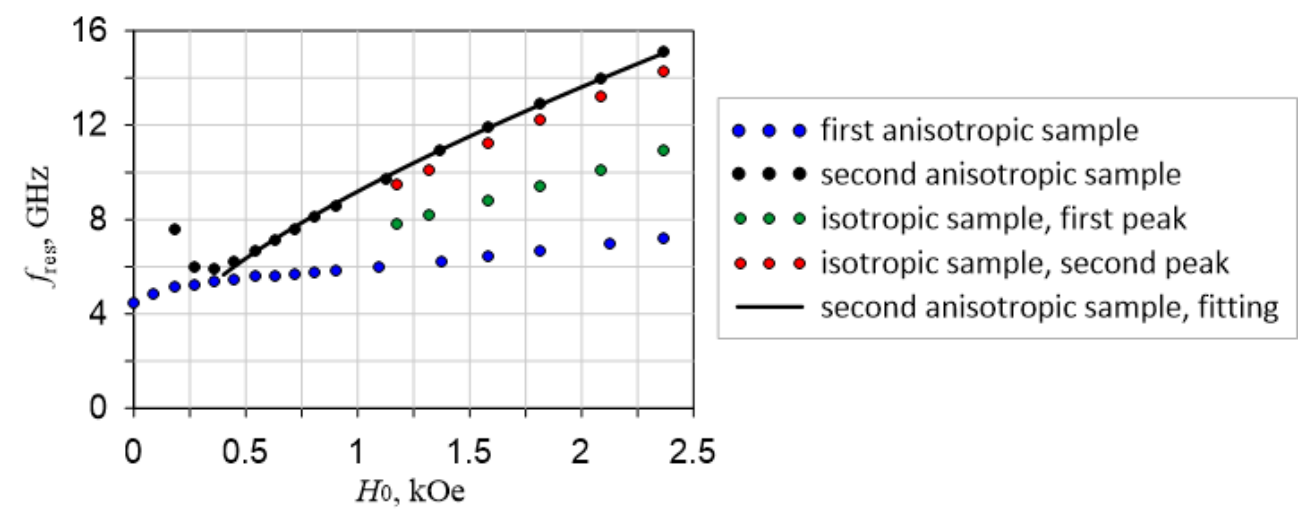

Figure 9. Dependence of the resonant frequency on the applied external field. The dots correspond to the measured data, and the black continuous curve corresponds to the fitting by Kittel's law.

The second anisotropic sample has a lower saturation field due to the small demagnetization factor. Kittel's rule may be used to find the magnetic characteristics of the material. The magnetic loss peak of the second anisotropic sample has a Lorentzian shape at fields more than 800 Oe (the dashed line in Figure 8). The dependence of the resonant frequency on the external field is shown in Figure 9 by the black circles. The dependence is fitted by Kittel's law (1), from which the saturation magnetization and the anisotropy field are found. The discrepancy between the fitting and the measured data is observed because the sample is not saturated at low fields. The found saturation magnetization is $4 \pi M_{0}=0.9 \mathrm{~T}$ and is close to the values from the archived literature $(1.0 \mathrm{~T})$. Although the particles in the sample are ordered, the anisotropy fields are randomly oriented in the particles' plane. Because of this, the average value of the anisotropy field is close to zero. Therefore, the found value of the anisotropy field $(5.8 \mathrm{Oe})$ is small. The accordance of the permeability to Kittel's theory and the equality of the found magnetic characteristics with the reference values confirms that the magnetic loss peak is associated with FMR and the demagnetizing effects do not influence the measurements.

\section{Conclusions}

The permeability of composites filled with flake sendust (Fe-Si-Al alloy) particles is measured as a function of frequency and external magnetic field. It is found that the magnetic loss peak is split under external fields of more than $1.5 \mathrm{kOe}$. To study this effect, the samples of various thicknesses and the samples with isotropic and anisotropic orientations of particles are measured. The particles in the anisotropic samples are oriented by a strong uniform magnetic field. At a small fraction of inclusions, the permanent magnetic field is demagnetized on the individual particles rather than the whole sample. The splitting of the magnetic loss peak of the isotropic sample is caused by a different orientation of particles in the sample. At a high fraction of inclusions, the permanent magnetic field is demagnetized on the whole sample, and the magnetic loss peak of the isotropic sample is not split. The saturation magnetization of the material is found by measurements under an external magnetic field of the anisotropic sample.

Author Contributions: Conceptualization, A.S. and K.R.; methodology, A.S. and D.P.; software, D.P.; formal analysis, A.S. and K.R.; investigation, A.S., A.N., A.A., S.M. and S.B.; writing-original draft preparation, A.S.; writing - review and editing, A.S. and K.R. All authors have read and agreed to the published version of the manuscript.

Funding: This research was funded by Russian Foundation for Basic Research (RFBR), project number 19-32-90165.

Data Availability Statement: Data supporting this article are available from the corresponding author upon reasonable request.

Conflicts of Interest: The authors declare no conflict of interest. 


\section{References}

1. Garner, A.; Parker, G.; Simone, D. Accounting for conducting inclusion permeability in the microwave regime in a modified generalized effective medium theory. IEEE Trans. Dielectr. Electr. Insul. 2015, 22, 2064-2072. [CrossRef]

2. Li, Q.; Chen, Y.; Harris, V. Clustering effect on permeability spectra of magneto-dielectric composites with conductive magnetic inclusions. J. Appl. Phys. 2019, 125, 185107. [CrossRef]

3. Han, M.; Rozanov, K.; Zezyulina, P.; Wu, Y. Effects of eddy current and dispersion of magnetic anisotropy on the high-frequency permeability of Fe based nanocomposites. J. Magn. Magn. Mater. 2015, 383, 114-119. [CrossRef]

4. Geyer, R.; Asadi-Zeydabadi, M. Tailored dielectric and magnetic properties of composite electroceramics with ferroelectric and ferrimagnetic components. J. Appl. Phys. 2018, 124, 164104. [CrossRef]

5. Kasagi, T.; Tsutaoka, T.; Hatakeyama, K. Electromagnetic properties of Permendur granular composite materials containing flaky particles. J. Appl. Phys. 2014, 116, 153901. [CrossRef]

6. Pardavi-Horvath, M. Microwave applications of soft ferrites. J. Magn. Magn. Mater. 2000, 215-216, 171-183. [CrossRef]

7. Salahun, E.; Queffelec, P.; Le Floc'h, M.; Gelin, P. A broadband permeameter for "in situ" measurements of rectangular samples. IEEE Trans. Magn. 2001, 37, 2743-2745. [CrossRef]

8. Cramer, N.; Lucic, D.; Camley, R.E.; Celinski, Z. High attenuation tunable microwave notch filters utilizing ferromagnetic resonance. J. Appl. Phys. 2000, 87, 6911-6913. [CrossRef]

9. Chevalier, A.; Le Floc'h, M. Dynamic permeability in soft magnetic composite materials. J. Appl. Phys. 2001, 90, 3462-3465. [CrossRef]

10. Schmool, D.S.; Apolinario, A.; Casoli, F.; Albertini, F. Ferromagnetic Resonance Study of Fe/FePt Coupled Films with Perpendicular Anisotropy. IEEE Trans. Magn. 2008, 40, 3087-3090. [CrossRef]

11. Tomita, S.; Hagiwara, M.; Kashiwagi, T.; Tsuruta, C.; Matsui, Y.; Fujii, M.; Hayashi, S. Ferromagnetic resonance study of diluted Fe nanogranular films. J. Appl. Phys. 2004, 95, 8194-8198. [CrossRef]

12. Maksymov, I.; Kostylev, M. Broadband stripline ferromagnetic resonance spectroscopy of ferromagnetic films, multilayers and nanostructures. Physica. E Low-Dimens. Syst. Nanostructures 2015, 69, 253-293. [CrossRef]

13. Shiryaev, A.O.; Rozanov, K.N.; Vyzulin, S.A.; Kevraletin, A.L.; Syr'ev, N.E.; Vyzulin, E.S.; Lahderanta, E.; Maklakov, S.A.; Granovsky, A.B. Magnetic resonances and microwave permeability in thin Fe films on flexible polymer substrates. J. Magn. Magn. Mater. 2018, 461, 76-81. [CrossRef]

14. Mattei, J.L.; Le Floc'h, M. A numerical approach of the inner demagnetizing effects in soft magnetic composites. J. Magn. Magn. Mater. 2000, 215-216, 589-591. [CrossRef]

15. Mattei, J.; Le Floc'h, M. Percolative behaviour and demagnetizing effects in disordered heterostructures. J. Magn. Magn. Mater. 2003, 257, 335-345. [CrossRef]

16. Mattei, J.; Le Floc'h, M. Effects of the magnetic dilution on the ferrimagnetic resonance of disordered hetero structures. J. Magn. Mag. Mater. 2003, 264, 86-94. [CrossRef]

17. Shiryaev, A.O.; Rozanov, K.N.; Starostenko, S.N.; Bobrovskii, S.Y.; Osipov, A.V.; Petrov, D.A. The bias effect on the frequency dispersion of microwave permeability of composites filled with metal films or flakes. J. Magn. Magn. Mater. 2019, 470, 139-142. [CrossRef]

18. Shiryaev, A.O.; Bobrovskii, S.Y.; Granovsky, A.B.; Osipov, A.V.; Naboko, A.S.; Lahderanta, E.; Lagarkov, A.N.; Rozanov, K.N.; Zezyulina, P.A. Coaxial measurements of microwave permeability of thin supermalloy films under magnetic bias. JMMM 2019, 477, 329-333. [CrossRef]

19. Starostenko, S.N.; Rozanov, K.N.; Shiryaev, A.O.; Shalygin, A.N.; Lagarkov, A.N. Determination of sendust intrinsic permeability from microwave constitutive parameters of composites with sendust spheres and flakes. J. Appl. Phys. 2017, 121, 245107. [CrossRef]

20. Chen, L.F.; Ong, C.K.; Neo, C.P.; Varadan, V.V.; Varadan, V.K. Microwave Electronics: Measurement and Materials Characterization; John Wiley \& Sons Ltd.: West Sussex, UK, 2004.

21. Rozanov, K.; Koledintseva, M. Analytical representations for frequency dependences of microwave permeability. In Proceedings of the 2012 IEEE International Symposium on Electromagnetic Compatibility, Pittsburgh, PA, USA, 5-10 August 2012. Abstract Number 13116484.

22. Fredkin, D.R.; Ron, A. Microscopic derivation of the Landau-Lifschitz equation for ferromagnetic relaxation. Phys. Rev. B 2000, 61, 8654-8655. [CrossRef]

23. Kittel, C. On the Theory of Ferromagnetic Resonance Absorption. Phys. Rev. 1948, 73, 155-161. [CrossRef]

24. Zhang, B.S.; Yuan, Z.; Zhao, H.; Yang, Y.; Tang, D.M. Hysteretic Behavior of the Dynamic Permeability in FeCoB Thin Films. IEEE Trans. Magn. 2016, 52, 2000204. [CrossRef]

25. Iakubov, I.T.; Kashurkin, O.Y.; Lagarkov, A.N.; Maklakov, S.A.; Osipov, A.V.; Rozanov, K.N.; Ryzhikov, I.A.; Starostenko, S.N. A contribution from the magnetoelastic effect to measured microwave permeability of thin ferromagnetic films. J. Magn. Magn. Mater. 2012, 324, 3385-3388. [CrossRef]

26. Chalapat, K.; Timonen, J.V.; Huuppola, M.; Koponen, L.; Johans, C.; Ras, R.H.; Ikkala, O.; Oksanen, M.A.; Seppälä, E.; Paraoanu, G.S. Ferromagnetic resonance in epsilon-Co magnetic composites. Nanotechnology 2014, 25, 485707. [CrossRef] [PubMed] 
27. Xu, X.; Jin, L.; Wen, T.; Liao, Y.; Tang, X.; Zhang, H.; Zhong, Z. Effects of substrate annealing on uniaxial magnetic anisotropy and ferromagnetic resonance frequency of Ni80Fe20 films deposited on self-organized periodically rippled sapphire substrates. Vacuum 2021, 186, 110047. [CrossRef]

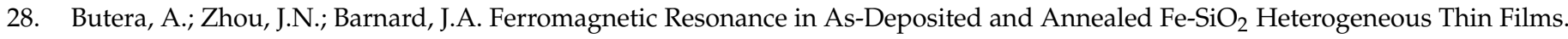
Phys. Rev. B 1999, 60, 12270-12278. [CrossRef]

29. Rozanov, K.N.; Koledintseva, M.Y. Application of generalized Snoek's law over a finite frequency range: A case study. J. Appl. Phys. 2016, 119, 073901. [CrossRef]

30. Nakamura, T.; Tsutaoka, T.; Hatakeyama, K. Frequency dispersion of permeability in ferrite composite materials. J. Magn. Magn. Mater. 1994, 138, 319-328. [CrossRef]

31. Neo, C.P.; Yang, Y.; Ding, J. Calculation of complex permeability of magnetic composite materials using ferromagnetic resonance model. J. Appl. Phys. 2010, 107, 083906. [CrossRef]

32. Tsutaoka, T. Frequency dispersion of complex permeability in $\mathrm{Mn}-\mathrm{Zn}$ and $\mathrm{Ni}-\mathrm{Zn}$ spinel ferrites and their composite materials. J. Appl. Phys. 2003, 93, 2789-2796. [CrossRef]

33. Starostenko, S.; Rozanov, K. Microwave Screen with Magnetically Controlled Attenuation. PIER 2009, 99, 405-426. [CrossRef]

34. Petrov, D.; Rozanov, N.; Koledintseva, M. Influence of Higher-order Modes in Coaxial Waveguide on Measurements of Material Parameters. In Proceedings of the 2018 IEEE Symposium on Electromagnetic Compatibility, Signal Integrity and Power Integrity, Long Beach, CA, USA, 30 July-3 August 2018.

35. Acher, O.; Adenot, A.L. Bounds on the dynamic properties of magnetic materials. Phys. Rev. B 2000, 62, 11324-11327. [CrossRef]

36. He, D.; Dou, Z.; Zhang, J.; Wang, P.; Wang, G.; Duan, B.; Qiao, L.; Wang, T. Effects of orientation methods on electromagnetic parameters and microwave absorption characteristics of flaky carbonyl iron $\mathrm{SiO}_{2}$ particles. J. Magn. Magn. Mater. 2020, 513, 167191. [CrossRef] 